PROCEEDINGS OF THE

AMERICAN MATHEMATICAL SOCIETY

Volume 140, Number 5, May 2012, Pages 1815-1822

S 0002-9939(2011)11036-0

Article electronically published on September 12, 2011

\title{
A LOCALIZATION FORMULA IN DIRICHLET FORM THEORY
}

\author{
ZHEN-QING CHEN AND MASATOSHI FUKUSHIMA
}

(Communicated by Richard C. Bradley)

\begin{abstract}
A localization energy formula is established for symmetric Markov processes on Luzin spaces.
\end{abstract}

\section{INTRODUCTION}

Let $E$ be a Luzin space, $m$ a $\sigma$-finite measure on it and $X$ an $m$-symmetric right process on $E$. Let $(\mathcal{E}, \mathcal{F})$ be the Dirichlet form on $L^{2}(E ; m)$ associated with $X$, which is known to be quasi regular. In view of the quasi homeomorphism method in [3, without loss of generality, we may and do assume that $E$ is a locally compact separable metric space, $m$ is a positive Radon measure on $E$ with $\operatorname{supp}[m]=E$, $(\mathcal{E}, \mathcal{F})$ is a regular symmetric Dirichlet form in $L^{2}(E ; m)$, and $X=\left(X_{t}, \mathbb{P}_{x}, \zeta\right)$ is an $m$-symmetric Hunt process associated with $(\mathcal{E}, \mathcal{F})$. We will use $\left(\mathcal{E}, \mathcal{F}_{e}\right)$ to denote the extended Dirichlet space of $(\mathcal{E}, \mathcal{F})$ and $\mathcal{E}_{1}:=\mathcal{E}+(\cdot, \cdot)_{L^{2}(E ; m)}$. The expectation with respect to the probability measure $\mathbb{P}_{x}$ will be denoted as $\mathbb{E}_{x}$. We will use the convention that any function defined on $E$ is extended to $E_{\partial}:=E \cup\{\partial\}$ by taking the value 0 at the cemetery point $\partial$ that is added to $E$ as a one-point compactification. Every element $u$ in $\mathcal{F}_{e}$ then admits a quasi continuous version and we will assume that functions in $\mathcal{F}_{e}$ are always represented by their quasi continuous versions. In the sequel, the abbreviations CAF, PCAF and MAF stands for "continuous additive functional", "positive continuous additive functional" and "martingale additive functional", respectively, whose definitions can be found both in [1] and [4. We also refer readers to the above two books for notions such as $m$-polar and $\mathcal{E}$-quasi everywhere $(\mathcal{E}$-q.e. in abbreviation).

Consider a Lévy system $(N(x, d y), H)$ for the $m$-symmetric Hunt process $X$ on $E$. The Revuz measure of the PCAF $H$ of $X$ will be denoted as $\mu_{H}$. We define

$$
J(d x, d y)=N(x, d y) \mu_{H}(d x) \quad \text { and } \quad \kappa(d x)=N(x,\{\partial\}) \mu_{H}(d x)
$$

as the jumping measure and the killing measure of $X$ (or, equivalently, of $(\mathcal{E}, \mathcal{F})$ ). For square-integrable martingales $M$ and $N$, we use $[M]$ to denote the quadratic

Received by the editors December 15, 2010 and, in revised form, January 26, 2011.

2010 Mathematics Subject Classification. Primary 60J25, 31C25; Secondary 60J45, 60J50.

Key words and phrases. Symmetric Markov process, Hunt process, Dirichlet form, extended Dirichlet space, energy measure, additive functional, martingale additive functional.

The first author's research was partially supported by NSF Grants DMS-0906743 and DMR1035196.

The second author's research was supported by Grant-in-Aid for Scientific Research of MEXT No. 19540125. 
variation process of $M$, and define their quadratic covariation process $[M, N]$ by $([M+N]-[M-N]) / 4$. The dual predictable projections of $[M]$ and $[M, N]$ are denoted as $\langle M\rangle$ and $\langle M, N\rangle$, respectively (cf. [5]). For $u \in \mathcal{F}_{e}$, the following Fukushima decomposition holds:

$$
u\left(X_{t}\right)-u\left(X_{0}\right)=M_{t}^{[u]}+N_{t}^{[u]}, \quad t \geq 0,
$$

where $M^{[u]}$ is an MAF of $X$ of finite energy and $N^{[u]}$ is a CAF of $X$ having zero energy. Let $M^{[u], c}$ be the continuous martingale part of $M^{[u]} .\left\langle M^{[u]}\right\rangle$ and $\left\langle M^{[u], c}\right\rangle$ are then PCAFs of $X$. We use $\mu_{\langle u\rangle}$ and $\mu_{\langle u\rangle}^{c}$ to denote their Revuz measures on $E$, respectively. Let $\left\{P_{t}, t \geq 0\right\}$ be the transition semigroup of $X$.

The following facts are well known (see [1, Chapter 4] or [4, Chapter 5]): For $u \in L^{2}(E ; m), u \in \mathcal{F}$ if and only if $\sup _{t>0} \frac{1}{t}\left(u-P_{t} u, u\right)_{L^{2}(E ; m)}<\infty$, and for $u \in \mathcal{F}$,

$$
\begin{aligned}
& \mathcal{E}(u, u)=\lim _{t \rightarrow 0} \frac{1}{t}\left(u-P_{t} u, u\right)_{L^{2}(E ; m)} \\
& =\lim _{t \rightarrow 0} \frac{1}{2 t} \mathbb{E}_{m}\left[\left(u\left(X_{t}\right)-u\left(X_{0}\right)\right)^{2}\right]+\lim _{t \rightarrow 0} \frac{1}{2 t} \int_{E} u(x)^{2}\left(1-P_{t} 1(x)\right) m(d x) .
\end{aligned}
$$

Moreover, for $u \in \mathcal{F}_{e}$,

$$
\begin{aligned}
\mu_{\langle u\rangle}(d x) & =\mu_{\langle u\rangle}^{c}(d x)+\left(\int_{E_{\partial}}(u(x)-u(y))^{2} N(x, d y)\right) \mu_{H}(d x) \\
& =\mu_{\langle u\rangle}^{c}(d x)+\int_{E}(u(x)-u(y))^{2} J(d x, d y)+u(x)^{2} \kappa(d x), \\
& \lim _{t \rightarrow 0} \frac{1}{t} \int_{E} u(x)^{2}\left(1-P_{t} 1(x)\right) m(d x)=\int_{E} u(x)^{2} \kappa(d x),
\end{aligned}
$$

and

$$
\begin{aligned}
\lim _{t \rightarrow 0} \frac{1}{t} \mathbb{E}_{m}\left[\left(u\left(X_{t}\right)-u\left(X_{0}\right)\right)^{2}\right]= & \mu_{\langle u\rangle}^{c}(E)+\int_{E \times E}(u(x)-u(y))^{2} J(d x, d y) \\
& +\int_{E} u(x)^{2} \kappa(d x) .
\end{aligned}
$$

It follows from (1.4) and (1.5) that for $u \in \mathcal{F}_{e}$,

$$
\lim _{t \rightarrow 0} \frac{1}{t} \mathbb{E}_{m}\left[\left(u\left(X_{t}\right)-u\left(X_{0}\right)\right)^{2} ; t<\zeta\right]=\mu_{\langle u\rangle}^{c}(E)+\int_{E \times E}(u(x)-u(y))^{2} J(d x, d y) .
$$

In addition, the following Beurling-Deny decomposition holds for the Dirichlet form $(\mathcal{E}, \mathcal{F})$ :

$\mathcal{E}(u, u)=\frac{1}{2} \mu_{\langle u\rangle}^{c}(E)+\frac{1}{2} \int_{E \times E}(u(x)-u(y))^{2} J(d x, d y)+\int_{E} u(x)^{2} \kappa(d x) \quad$ for $u \in \mathcal{F}_{e}$.

The above Beurling-Deny decomposition can be regarded as the analogy to symmetric Markov processes of the Lévy-Khinchin formula for Lévy processes. It characterizes the continuous part, the pure jumping part and the killings of the strong Markov process $X$. 
Let $D$ be a quasi open subset of $E$ and $X^{D}$ the subprocess of $X$ killed upon leaving $D$, The subprocess $X^{D}$ is then symmetric with respect to the measure $m_{0}:=\left.m\right|_{D}$. The Dirichlet form of $X^{D}$ is $\left(\mathcal{E}, \mathcal{F}^{D}\right)$, where

$$
\mathcal{F}^{D}=\left\{u \in \mathcal{F}: u=0 \mathcal{E} \text {-q.e. on } D^{c}\right\} .
$$

Let $\left\{P_{t}^{D}, t \geq 0\right\}$ denote the transition semigroup of $X^{D}$. For $u \in \mathcal{F}_{e}$, (1.2) tells us that neither $\lim _{t \rightarrow 0} \frac{1}{t} \int_{D} u(x)^{2}\left(1-P_{t}^{D} 1(x)\right) m_{0}(d x)$ nor $\lim _{t \rightarrow 0} \frac{1}{t} \mathbb{E}_{m_{0}}\left[\left(u\left(X_{t}^{D}\right)-\right.\right.$ $\left.\left.u\left(X_{0}^{D}\right)\right)^{2}\right]$ can be expected to exist in general unless $E \backslash D$ is $m$-polar. Nevertheless, the following main result of this paper asserts that (1.6) remains valid for $u \in \mathcal{F}_{e}$ with $\tau_{D}$ and $D$ in place of $\zeta$ and $E$, respectively.

Theorem 1.1. For every $v \in \mathcal{F}_{e}$,

$$
\lim _{t \rightarrow 0} \frac{1}{t} \mathbb{E}_{m_{0}}\left[\left(v\left(X_{t}\right)-v\left(X_{0}\right)\right)^{2} ; t<\tau_{D}\right]=\mu_{\langle v\rangle}^{c}(D)+\int_{D \times D}(v(x)-v(y))^{2} J(d x, d y) .
$$

It is a bit surprising to us that this localization formula was not known until now. Such a formula is very useful in the study of boundary theory of symmetric Markov processes, especially in deriving the Beurling-Deny decomposition of the Dirichlet form of the trace (the time-changed process) of the symmetric Markov process $X$ on a quasi closed set $F=E \backslash D$; see Section 5.5 and Section 5.6 of [1. This Beurling-Deny decomposition asserts (see [1, Theorem 5.5.9 and Corollary 5.6.1]) that for every $u \in \mathcal{F}_{e}$,

$$
\begin{aligned}
\mathcal{E}(\mathbf{H} u, \mathbf{H} u)= & \frac{1}{2} \mu_{\langle\mathbf{H} u\rangle}^{c}(F)+\frac{1}{2} \int_{F \times F}(u(x)-u(y))^{2}(J(d x, d y)+U(d x, d y)) \\
& +\int_{F} u(x)^{2}(\kappa(d x)+V(d x)),
\end{aligned}
$$

where $\mathbf{H} u(x)=\mathbb{E}_{x}\left[u\left(X_{\sigma_{F}}\right)\right], \sigma_{F}=\inf \left\{t>0: X_{t} \in F\right\}, U$ and $V$ are Feller measures of $X$ that characterize the excursions of $X$ around $F$. The identity (1.8) was first established in [2]. However, the proof of a key step, Theorem 2.6 of [2], while its conclusion is correct, contains a serious gap in that a dual predictable projection result was applied incorrectly on line 1 of p.1069 there. The use of Theorem[1.1enables us to give a correct proof of [2, Theorem 2.6]; see Theorem 5.5.8 of [1].

\section{Proof}

For notational convenience, let $F:=E \backslash D$ and $F_{\partial}:=F \cup\{\partial\}$.

Lemma 2.1. Suppose $v$ is a bounded function in $\mathcal{F}_{e}$. Then

$\limsup _{t \rightarrow 0} \frac{1}{t} \mathbb{E}_{m_{0}}\left[\left(v\left(X_{t}\right)-v\left(X_{0}\right)\right)^{2} ; t<\tau_{D}\right] \leq \mu_{\langle v\rangle}^{c}(D)+\int_{D \times D}(v(x)-v(y))^{2} J(d x, d y)$.

Proof. First note that

$$
\mathbb{E}_{x}\left[\left(M_{t \wedge \tau_{D}}^{[v]}\right)^{2}\right]=\mathbb{E}_{x}\left[\left\langle M^{[v]}\right\rangle_{t \wedge \tau_{D}}\right] .
$$

By [1, Proposition 4.1.10], $t \rightarrow\left\langle M^{[v]}\right\rangle_{t \wedge \tau_{D}}$ is a PCAF of $X^{D}$ and

$$
\begin{aligned}
& \lim _{t \rightarrow 0} \frac{1}{t} \mathbb{E}_{m_{0}}\left[\left\langle M^{[v]}\right\rangle_{t \wedge \tau_{D}}\right]=\mu_{\langle v\rangle}(D) \\
& =\mu_{\langle v\rangle}^{c}(D)+\int_{D \times E_{\partial}}(v(x)-v(y))^{2} N(x, d y) \mu_{H}(d x) .
\end{aligned}
$$


Define, for $t \geq 0, A_{t}:=\left(v\left(X_{\tau_{D}}\right)-v\left(X_{\tau_{D^{-}}}\right)\right) \mathbf{1}_{\left\{t \geq \tau_{D}>0\right\}}$, and let $A^{p}$ be its dual predictable projection. Since $A$ is a process of bounded variation, $A^{p}$ can be expressed as

$$
A_{t}^{p}=\int_{0}^{t \wedge \tau_{D}} \int_{F_{\partial}}\left(v(y)-v\left(X_{s}\right)\right) N\left(X_{s}, d y\right) d H_{s},
$$

on account of the Lévy system formula (see, e.g., [1, (A.3.33)]. It is known (see, e.g., [5]) that $M:=A-A^{p}$ is a purely discontinuous square integrable martingale that is orthogonal to $M_{\cdot \wedge \tau_{D}}^{[v]}-M$ in the sense that $\left[M, M_{\cdot \wedge \tau_{D}}^{[v]}-M\right]=0$. We claim that

$$
\lim _{t \rightarrow 0} \frac{1}{t} \mathbb{E}_{m_{0}}\left[\left(A_{t}^{p}\right)^{2}\right]=0 .
$$

To prove it, for $k \geq 1$, define

$$
A_{t}^{k}:=\left(v\left(X_{\tau_{D}}\right)-v\left(X_{\tau_{D}-}\right)\right) \mathbf{1}_{\left\{\left|v\left(X_{\tau_{D}}\right)-v\left(X_{\tau_{D^{-}}}\right)\right|>1 / k\right\}} \mathbf{1}_{\left\{t \geq \tau_{D}>0\right\}}
$$

and

$$
A_{t}^{k, p}:=\int_{0}^{t \wedge \tau_{D}} \int_{F_{\partial}}\left(v(y)-v\left(X_{s}\right)\right) \mathbf{1}_{\left\{\left|v(y)-v\left(X_{s}\right)\right|>1 / k\right\}} N\left(X_{s}, d y\right) d H_{s} .
$$

Then $M^{k}:=A^{k}-A^{k, p}$ is a purely discontinuous square integrable martingale and $\left[M-M^{k}\right]_{t}=\left(A_{t}-A_{t}^{k}\right)^{2}$. Therefore by the Lévy system formula mentioned above,

$$
\begin{aligned}
& \limsup _{t \rightarrow 0} \frac{1}{t} \mathbb{E}_{m_{0}}\left[\left(A_{t}^{p}-A_{t}^{k, p}\right)^{2}\right] \\
& \quad \leq \limsup _{t \rightarrow 0} \frac{2}{t} \mathbb{E}_{m_{0}}\left[\left(M_{t}-M_{t}^{k}\right)^{2}\right]+\limsup _{t \rightarrow 0} \frac{2}{t} \mathbb{E}_{m_{0}}\left[\left(A_{t}-A_{t}^{k}\right)^{2}\right] \\
& \quad \leq 4 \int_{D \times F_{\partial}}(v(x)-v(y))^{2} \mathbf{1}_{\{|v(x)-v(y)| \leq 1 / k\}} J(d x, d y),
\end{aligned}
$$

which tends to 0 as $k \rightarrow \infty$. Now define

$$
B_{t}^{k}:=\left|v\left(X_{\tau_{D}}\right)-v\left(X_{\tau_{D}-}\right)\right| \mathbf{1}_{\left\{\left|v\left(X_{\tau_{D}}\right)-v\left(X_{\tau_{D^{-}}}\right)\right|>1 / k\right\}} \mathbf{1}_{\left\{t \geq \tau_{D}>0\right\}}
$$

and

$$
B_{t}^{k, p}:=\int_{0}^{t \wedge \tau_{D}} \int_{F_{\partial}}\left|v(y)-v\left(X_{s}\right)\right| \mathbf{1}_{\left\{\left|v(y)-v\left(X_{s}\right)\right|>1 / k\right\}} N\left(X_{s}, d y\right) d H_{s} .
$$

Then

$$
\mathbb{E}_{x}\left[B_{t}^{k, p}\right]=\mathbb{E}_{x}\left[B_{t}^{k}\right] \leq 2\|v\|_{\infty} \mathbb{P}_{x}\left(t \geq \tau_{D}\right) \quad \text { for } x \in D
$$

and $B^{k, p}$ is a PCAF of $X^{D}$ having Revuz measure $\mu_{k}$ with

$$
\begin{aligned}
\mu_{k}(D) & =\int_{D \times F_{\partial}}|v(x)-v(y)| \mathbf{1}_{\{|v(x)-v(y)|>1 / k\}} N(x, d y) \mu_{H}(d x) \\
& \leq k \int_{D \times F_{\partial}}(v(x)-v(y))^{2} N(x, d y) \mu_{H}(d x)<\infty .
\end{aligned}
$$

By the Markov property of $X^{D}$, equation (2.5) and Revuz correspondence (see, e.g., [1, Theorem 4.1.1]), 


$$
\begin{aligned}
& \mathbb{E}_{m_{0}}\left[\left(A_{t}^{k, p}\right)^{2}\right] \leq \mathbb{E}_{m_{0}}\left[\left(B_{t}^{k, p}\right)^{2}\right]=2 \mathbb{E}_{m_{0}}\left[\int_{0}^{t}\left(\int_{s}^{t} d B_{r}^{k, p}\right) d B_{s}^{k, p}\right] \\
& =2 \mathbb{E}_{m_{0}}\left[\int_{0}^{t} \mathbb{E}_{X_{s}^{D}}\left[B_{t-s}^{k, p}\right] d B_{s}^{k, p}\right] \leq 4\|v\|_{\infty} \mathbb{E}_{m_{0}}\left[\int_{0}^{t}\left(1-P_{t-s}^{D} 1\left(X_{s}^{D}\right)\right) d B_{s}^{k, p}\right] \\
& \leq 4\|v\|_{\infty} \int_{0}^{t}\left(\left\langle P_{s}^{D} 1, \mu_{k}\right\rangle-\left\langle P_{s}^{D} 1, P_{t}^{D} 1 \cdot \mu_{k}\right\rangle\right) d s .
\end{aligned}
$$

It then follows from the dominated convergence theorem that

$$
\limsup _{t \rightarrow 0} \frac{1}{t} \mathbb{E}_{m_{0}}\left[\left(A_{t}^{k, p}\right)^{2}\right] \leq 4\|v\|_{\infty}\left(\mu_{k}(D)-\mu_{k}(D)\right)=0 .
$$

This together with (2.4) establishes the claim (2.3).

Next by Fukushima's decomposition, (2.3), the stated martingale orthogonality between $M$ and $M_{\cdot \wedge \tau_{D}}^{[v]}-M$, the identity $[M]_{t}=A_{t}^{2}$ and finally by (2.1) and the Lévy system formula, we have

$$
\begin{aligned}
& \limsup _{t \rightarrow 0} \frac{1}{t} \mathbb{E}_{m_{0}}\left[\left(v\left(X_{t}\right)-v\left(X_{0}\right)\right)^{2} ; t<\tau_{D}\right]=\limsup _{t \rightarrow 0} \frac{1}{t} \mathbb{E}_{m_{0}}\left[\left(M_{t \wedge \tau_{D}}^{[v]}\right)^{2} ; t<\tau_{D}\right] \\
& =\limsup _{t \rightarrow 0} \frac{1}{t} \mathbb{E}_{m_{0}}\left[\left(M_{t \wedge \tau_{D}}^{[v]}-M_{t}-A_{t}^{p}\right)^{2} ; t<\tau_{D}\right] \\
& \leq \underset{t \rightarrow 0}{\limsup } \frac{1}{t} \mathbb{E}_{m_{0}}\left[\left(M_{t \wedge \tau_{D}}^{[v]}-M_{t}\right)^{2}\right] \\
& =\limsup _{t \rightarrow 0} \frac{1}{t} \mathbb{E}_{m_{0}}\left[\left(M_{t \wedge \tau_{D}}^{[v]}\right)^{2}\right]-\lim _{t \rightarrow 0} \frac{1}{t} \mathbb{E}_{m_{0}}\left[A_{t}^{2}\right] \\
& =\mu_{\langle v\rangle}^{c}(D)+\int_{D \times D}(v(x)-v(y))^{2} N(x, d y) \mu_{H}(d x) .
\end{aligned}
$$

This completes the proof of the lemma.

Proof of Theorem 1.1. It suffices to prove the theorem for $v=R_{\alpha} g$ for some bounded $g \in L^{2}(E ; m)$, as such functions $v$ are $\mathcal{E}$-dense in $\mathcal{F}_{e}$ and the upper bound in Lemma 2.1 can be utilized.

For $f \in \mathcal{F}^{D} \subset \mathcal{F}$, let the Fukushima decomposition of $f\left(X_{t}^{D}\right)-f\left(X_{0}^{D}\right)$ be denoted as $M_{t}^{0,[f]}+N_{t}^{0,[f]}$, while the Fukushima decomposition for $f\left(X_{t}\right)-f\left(X_{0}\right)$ is denoted by $M_{t}^{[f]}+N_{t}^{[f]}$. Since $f\left(X_{t \wedge \tau_{D}}\right)-f\left(X_{0}\right)=f\left(X_{t}^{D}\right)-f\left(X_{0}^{D}\right)$, we have

$$
M_{t \wedge \tau_{D}}^{[f]}-M_{t}^{0,[f]}=N_{t}^{0,[f]}-N_{t \wedge \tau_{D}}^{[f]}, \quad t \geq 0 .
$$

It is easy to check (see [1, Exercise 4.1.9]) that $M_{t}^{0,[f]}$ is a square-integrable martingale with respect to the filtration $\left\{\mathcal{F}_{t \wedge \tau_{D}}, t \geq 0\right\}$ and so is $M_{t \wedge \tau_{D}}^{[f]}-M_{t}^{0,[f]}$. Since $N^{[f]}$ (resp. $\left.N^{0,[f]}\right)$ is a CAF of $X\left(\right.$ resp. $\left.X^{D}\right)$ of zero energy, we have

$$
\begin{aligned}
& \mathbb{E}_{m_{0}}\left[\left\langle M_{\cdot \wedge \tau_{D}}^{[f]}-M^{0,[f]}\right\rangle_{t} ; t<\tau_{D}\right]=\mathbb{E}_{m_{0}}\left[\left\langle N_{\cdot \wedge \tau_{D}}^{[f]}-N^{0,[f]}\right\rangle_{t} ; t<\tau_{D}\right] \\
& =\mathbb{E}_{m_{0}}\left[\lim _{n \rightarrow \infty} \sum_{k=1}^{n}\left(N_{k t / n}^{[f]}-N_{(k-1) t / n}^{[f]}-N_{k t / n}^{0,[f]}+N_{(k-1) t / n}^{0,[f]}\right)^{2} ; t<\tau_{D}\right] \\
& \leq \lim _{n \rightarrow \infty} 2 \mathbb{E}_{m}\left[\sum_{k=1}^{n}\left(N_{k t / n}^{[f]}-N_{(k-1) t / n}^{[f]}\right)^{2}\right] \\
& \quad+\lim _{n \rightarrow \infty} 2 \mathbb{E}_{m_{0}}\left[\sum_{k=1}^{n}\left(N_{k t / n}^{0,[f]}-N_{(k-1) t / n}^{0,[f]}\right)^{2}\right]=0 .
\end{aligned}
$$


By the continuity of $\left\langle M_{\cdot \wedge \tau_{D}}^{[f]}-M^{0,[f]}\right\rangle_{t}$, we conclude that $\left\langle M_{\cdot \wedge \tau_{D}}^{[f]}-M^{0,[f]}\right\rangle_{\tau_{D}}=0$ and therefore $M_{t \wedge \tau_{D}}^{[f]}=M_{t}^{0,[f]}$. Consequently, $N_{t \wedge \tau_{D}}^{[f]}=N_{t}^{0,[f]}$.

Now let $f=\alpha R_{\alpha}^{D} \mathbf{1}_{D \cap K} \in \mathcal{F}^{D}$ for a fixed compact set $K \subset E$. Note that $0 \leq f \leq 1$. By Fukushima's decomposition and the fact that $t \mapsto\left\langle M^{[v]}\right\rangle_{t \wedge \tau_{D}}$ is a PCAF of $X^{D}$ with Revuz measure $\left.\mu_{\langle v\rangle}\right|_{D}$ (see Proposition 4.1 .10 of [1]),

$$
\begin{aligned}
& \lim _{t \rightarrow 0} \frac{1}{t} \mathbb{E}_{m_{0}}\left[\left(v\left(X_{t}\right)-v\left(X_{0}\right)\right)^{2} ; t<\tau_{D}\right] \\
& =\lim _{t \rightarrow 0} \frac{1}{t} \mathbb{E}_{m_{0}}\left[\left(M_{t \wedge \tau_{D}}^{v}\right)^{2} ; t<\tau_{D}\right] \geq \lim _{t \rightarrow 0} \frac{1}{t} \mathbb{E}_{m_{0}}\left[\left(M_{t \wedge \tau_{D}}^{[v]}\right)^{2} f\left(X_{t}^{D}\right)\right] \\
& =\lim _{t \rightarrow 0} \frac{1}{t} \mathbb{E}_{f \cdot m_{0}}\left[\left(M_{t \wedge \tau_{D}}^{[v]}\right)^{2}\right]+\lim _{t \rightarrow 0} \frac{1}{t} \mathbb{E}_{m_{0}}\left[\left(M_{t \wedge \tau_{D}}^{[v]}\right)^{2}\left(f\left(X_{t}^{D}\right)-f\left(X_{0}^{D}\right)\right)\right] \\
& =\lim _{t \rightarrow 0} \frac{1}{t} \mathbb{E}_{f \cdot m_{0}}\left[\left\langle M^{[v]}\right\rangle_{t \wedge \tau_{D}}\right]+\lim _{t \rightarrow 0} \frac{1}{t} \mathbb{E}_{m_{0}}\left[\left(M_{t \wedge \tau_{D}}^{[v]}\right)^{2}\left(f\left(X_{t \wedge \tau_{D}}\right)-f\left(X_{0}\right)\right)\right] \\
(2.6) & =\int_{D} f(x) \mu_{\langle v\rangle}(d x)+\lim _{t \rightarrow 0} \frac{1}{t} \mathbb{E}_{m_{0}}\left[\left(M_{t \wedge \tau_{D}}^{[v]}\right)^{2} M_{t \wedge \tau_{D}}^{[f]}\right]=: \int_{D} f(x) \mu_{\langle v\rangle}(d x)+I .
\end{aligned}
$$

In the second to last equality, we used the fact that

$$
N_{t \wedge \tau_{D}}^{[f]}=N_{t}^{0,[f]}=\int_{0}^{t \wedge \tau_{D}} \alpha\left(f-\mathbf{1}_{D \cap K}\right)\left(X_{s}\right) d s
$$

whose absolute value is bounded by $\alpha$ t. By Itô's formula,

$$
\begin{aligned}
I= & \lim _{t \rightarrow 0} \frac{1}{t} \mathbb{E}_{m_{0}}\left[\int_{0}^{t \wedge \tau_{D}} M_{s-}^{v} d\left\langle M^{[v], c} M^{[f], c}\right\rangle_{s}\right. \\
& \left.+\sum_{s \leq t \wedge \tau_{D}}\left(\left(M_{s}^{[v]}\right)^{2}-\left(M_{s-}^{[v]}\right)^{2}\right)\left(M_{s}^{[f]}-M_{s-}^{[f]}\right)\right] \\
= & \lim _{t \rightarrow 0} \frac{1}{t} \mathbb{E}_{m_{0}}\left[\int_{0}^{t \wedge \tau_{D}} M_{s-}^{v} d\left\langle M^{[v], c} M^{[f], c}\right\rangle_{s}\right. \\
& +\sum_{s \leq t \wedge \tau_{D}} 2 M_{s-}^{[v]}\left(M_{s}^{[v]}-M_{s-}^{[v]}\right)\left(M_{s}^{[f]}-M_{s-}^{[f]}\right) \\
& \left.+\sum_{s \leq t \wedge \tau_{D}}\left(M_{s}^{[v]}-M_{s-}^{[v]}\right)^{2}\left(M_{s}^{[f]}-M_{s-}^{[f]}\right)\right] .
\end{aligned}
$$

Since $v=R_{\alpha} g$ for some bounded $g \in L^{2}(E ; m)$,

$$
M_{t}^{[v]}=v\left(X_{t}\right)-v\left(X_{0}\right)-\int_{0}^{t}(\alpha u-g)\left(X_{s}\right) d s
$$

Observe that $\|\alpha u-g\|_{\infty} \leq 2\|g\|_{\infty}$ and so $\left|\int_{0}^{t}(\alpha u-g)\left(X_{s}\right) d s\right| \leq 2\|g\|_{\infty} t$. We then have by the Revuz formula in Proposition 4.1.10 of [1, the Lévy system formula, 


$$
\begin{aligned}
I= & \lim _{t \rightarrow 0} \frac{1}{t} \mathbb{E}_{m_{0}}\left[\int_{0}^{t \wedge \tau_{D}}\left(v\left(X_{s}\right)-v\left(X_{0}\right)\right) d\left\langle M^{[v], c} M^{[f], c}\right\rangle_{s}\right. \\
& +\sum_{s \leq t \wedge \tau_{D}} 2\left(v\left(X_{s-}\right)-v\left(X_{0}\right)\right)\left(v\left(X_{s}\right)-v\left(X_{s-}\right)\right)\left(f\left(X_{s}\right)-f\left(X_{s-}\right)\right) \\
& \left.+\sum_{s \leq t \wedge \tau_{D}}\left(v\left(X_{s}\right)-v\left(X_{s-}\right)\right)^{2}\left(f\left(X_{s}\right)-f\left(X_{s-}\right)\right)\right] \\
=0 & +\lim _{t \rightarrow 0} \frac{1}{t} \mathbb{E}_{m_{0}}\left[2 \int_{0}^{t \wedge \tau_{D}} v\left(X_{s}\right) \int_{E_{\partial}}\left(v\left(X_{s}\right)-v(y)\right)\left(f\left(X_{s}\right)-f(y)\right) N\left(X_{s}, d y\right) d H_{s}\right] \\
& \quad-\lim _{t \rightarrow 0} \frac{1}{t} \mathbb{E}_{v \cdot m_{0}}\left[2 \int_{0}^{t \wedge \tau_{D}} \int_{E_{\partial}}\left(v\left(X_{s}\right)-v(y)\right)\left(f\left(X_{s}\right)-f(y)\right) N\left(X_{s}, d y\right) d H_{s}\right] \\
& +\lim _{t \rightarrow 0} \frac{1}{t} \mathbb{E}_{m_{0}}\left[\int_{0}^{t \wedge \tau_{D}} \int_{E_{\partial}}\left(v(y)-v\left(X_{s}\right)\right)^{2}\left(f(y)-f\left(X_{s}\right)\right) N\left(X_{s}, d y\right) d H_{s}\right] \\
= & \int_{D \times E_{\partial}}(v(y)-v(x))^{2}(f(y)-f(x)) N(x, d y) d \mu_{H}(d x) \\
=- & \int_{D \times F_{\partial}} f(x)(v(x)-v(y))^{2} N(x, d y) d \mu_{H}(d x) .
\end{aligned}
$$

In the last equality above, we used the symmetry of $J$ and the fact that $f=0$ q.e. on $F$. Thus we have by (1.3) and (2.6),

$$
\begin{aligned}
\lim _{t \rightarrow 0} \frac{1}{t} \mathbb{E}_{m_{0}}\left[\left(v\left(X_{t}\right)-v\left(X_{0}\right)\right)^{2} ; t<\tau_{D}\right] \\
\quad \geq \int_{D} f(x) \mu_{\langle v\rangle}(d x)-\int_{D \times F_{\partial}} f(x)(v(x)-v(y))^{2} N(x, d y) d \mu_{H}(d x) \\
\quad=\int_{D} f(x) \mu_{\langle v\rangle}^{c}(d x)+\int_{D \times D} f(x)(v(x)-v(y))^{2} N(x, d y) d \mu_{H}(d x) .
\end{aligned}
$$

Since this is true for all $f=\alpha R_{\alpha}^{D} \mathbf{1}_{D \cap K}$, where $\alpha>0$ and $K$ is a compact subset of $E$, by first letting $K \uparrow E$ and then $\alpha \uparrow \infty$ we conclude that

$$
\begin{aligned}
\lim _{t \rightarrow 0} \frac{1}{t} \mathbb{E}_{m_{0}}\left[\left(v\left(X_{t}\right)-v\left(X_{0}\right)\right)^{2} ; t<\tau_{D}\right] \\
\quad \geq \mu_{\langle v\rangle}^{c}(D)+\int_{D \times D}(v(x)-v(y))^{2} N(x, d y) d \mu_{H}(d x) .
\end{aligned}
$$

This together with Lemma 2.1 completes the proof of the theorem.

\section{REFERENCES}

[1] Z.-Q. Chen and M. Fukushima, Symmetric Markov Processes, Time Change, and Boundary Theory. Princeton University Press, to appear.

[2] Z.-Q. Chen, M. Fukushima and J. Ying, Traces of symmetric Markov processes and their characterizations, Ann. Probab. 34 (2006), 1052-1102. MR2243879(2008a:60194)

[3] Z.-Q. Chen, Z.-M. Ma and M. Röckner, Quasi-homeomorphisms of Dirichlet forms. Nagoya Math. J. 136 (1994), 1-15. MR1309378 (95m:31020) 
[4] M. Fukushima, Y. Oshima and M. Takeda, Dirichlet Forms and Symmetric Markov Processes, Walter de Gruyter, 1994, 2nd rev. and ext. ed., 2010. MR.1303354 (96f:60126)

[5] S. W. He, J. G. Wang and J. A. Yan, Semimartingale Theory and Stochastic Calculus. Science Press, Beijing and New York, 1992. MR1219534 (95d:60080)

Department of Mathematics, University of Washington, Seattle, Washington 98195

E-mail address: zqchen@uw.edu

Branch of Mathematical Science, Osaka University, Toyonaka, Osaka 560-0043, JAPAN

E-mail address: fuku2@mx5.canvas.ne.jp 